\title{
ESTIMATION OF FIBRE ORIENTATION FROM DIGITAL IMAGES
}

\author{
SALME KÄRKKÄINEN ${ }^{1}$ AND EVA B VEDEL JENSEN ${ }^{2}$ \\ ${ }^{1}$ Department of Mathematics and Statistics, University of Jyväskylä, P.O. Box 35 (MaD), FIN-40351 Jyväskylä, \\ Finland, ${ }^{2}$ Laboratory for Computational Stochastics, Department of Mathematical Sciences, Ny Munkegade, \\ DK-8000 Aarhus C, Denmark \\ e-mail: samk@maths.jyu.fi,eva@imf.au.dk \\ (Accepted August 24, 2001)
}

\begin{abstract}
In this paper, estimation of fibre orientation is studied for fibre systems observable as a blurred greyscale image. The estimation method is based on scaled variograms observed along a set of sampling lines in different directions. The parameters of the orientation distribution are obtained numerically. Simulated data are used to study the statistical properties of the method.
\end{abstract}

Keywords: Boolean model, digitization, fibre orientation, image analysis, simulation, stereology.

\section{INTRODUCTION}

Fibre orientation is one of the important quality factors of fibrous materials affecting the physical properties of the material. Our objective is to estimate the orientation distribution from digital images of paper samples where the fibre structure is a blur and not visible. The fibres are described as a Boolean model of 'thick' fibres, $c f$. Matheron (1972), Mecke and Stoyan (1980) and Molchanov and Stoyan (1994), and the orientation distribution is modelled using the rose of directions of a fibre process (Mecke and Stoyan, 1980). The observed digital image is either binary or multicoloured. In the latter case, a digitized dead leaves model, $c f$. Jeulin (1989), possibly with additional blurring, is used to describe the image. The stereological estimation of the rose of directions is well-known when the counts of intersections between the fibre system and sampling lines in different directions can be observed, $c f$. Mecke and Stoyan (1980). In our case instead, the data consist of scaled variograms of grey values on the lines observed from the digital image without knowing the intersection counts. The stereological estimation method has been extended to the case of digital greyscale image data in Kärkkäinen et al. (2001b). Following Jeulin (2000) and Kärkkäinen et al. (2001a), an approximative proportionality between the expected counts of intersections and scaled variograms has been used in the estimation procedure together with a digitization correction. Assuming a parametric rose of directions this leads to nonlinear estimation equations and the parameters are obtained numerically. In order to further enlarge the applicability of this approach, especially for strongly anisotropic fibre processes, we suggest here an improvement to the proportionality model. This approach is applied to an image from a paper sample and simulated images. An alternative approach to the estimation of the orientation distribution from greyscale images can be found in Serra (1982). In the literature of paper manufacturing, the determination of fibre orientation is studied, e.g. in Forgacs and Strelis (1963), Paakkari et al. (1984), Hutten (1994), and in Erkkilä et al. (1998) where the method based on greyscale images is considered.

\section{THE IMAGE MODEL}

Fig. 1 (lower right) shows a digital video camera image of a layer extracted from a paper sample, $c f$. Erkkilä et al. (1998). The layer has been obtained from separation by a tape stripping technique. The underlying fibre system is degraded due to thickness, overlapping and optical properties of fibres, reflection of light and non-fibrous materials in the sample and also due to digitization.

We model the fibre system by a Boolean model (Matheron, 1972)

$$
\Phi=\cup_{n=1}^{\infty}\left(x_{n}+\Gamma_{n}\right),
$$

where $\left\{x_{n}\right\}$ is a stationary Poisson point process with intensity $\lambda$ and $\left\{\Gamma_{n}\right\}$ an i.i.d sequence of line segments with random length $l$ (mean $\bar{l}$ ) and with orientation distribution given by the density $f_{\mathscr{R}}$ of rose of directions $\mathscr{R}$. Furthermore, $\left\{\Gamma_{n}\right\}$ is independent of $\left\{x_{n}\right\}$. Then, the intensity of $\Phi$ is $L_{A}=\lambda \bar{l}$. In paper technology, $f_{\mathscr{R}}$ is often modelled by an elliptic distribution (Forgacs and Strelis, 1963)

$$
f_{\mathscr{R}}(\alpha ; \tau, \kappa)=\frac{c}{\sqrt{1-\kappa^{2} \cos ^{2}(\alpha-\tau)}}, \quad 0 \leq \alpha<\pi,
$$


where $c$ is the normalizing constant, the preferred direction of fibres $\tau \in[0, \pi)$ is orthogonal to the machine direction and the strength of anisotropy $\kappa \in$ $(0,1)$ describes deviation from the circular model $(\kappa=$ 0 ). A simulated realization from this model is shown in the upper left image of Fig. 1.

In practice, we observe a random field $\{Z(x)=$ $\left.h(x ; \Phi), x \in \mathbb{R}^{2}\right\}$, where $h$ is a complex unknown degradation function. In the simplest case, fibres are allowed to have thickness leading to $\left\{Z(x)=1_{\Xi}(x)\right\}$ with

$$
\Xi=\cup_{n=1}^{\infty}\left(x_{n}+\Xi_{n}\right) \text {. }
$$

Here $\Xi_{n}$ is a 'thick' version of $\Gamma_{n}$. In the upper middle of Fig. 1, a realization of a digitized version of $Z=1_{\Xi}$ is shown, with $\Xi_{n}$ equal to a flat ellipse with major axis $\Gamma_{n}$.

In more complex cases, a greyscale image is observed. We have here used a dead leaves model with finite time (Jeulin, 1989). Each point of the greyscale image has either the background colour or the colour of the fibre fallen most recently on that point. Blur has been modelled by moving average filtering. Used iteratively, it approximates Gaussian filtering (e.g. Glasbey and Horgan, 1995). Simulations are shown in the upper right and lower left image of Fig. 1.

\section{THE ESTIMATION METHOD}

The estimation of the orientation distribution $f_{\mathscr{R}}$ is based on stereological data. The image is intersected by lines in different directions and the variation of grey level is observed as a scaled variogram along each line. The estimation is based on a well-known formula, concerning intersections of the fibre process $\Phi$ and the sampling line $L_{\beta}$ at an angle $\beta \in[0, \pi)$ to the $x$-axis. The orientation distribution is related to the intensity $P_{L}^{0}(\beta)$ of the point process $L_{\beta} \cap \Phi$ according to

$$
P_{L}^{0}(\beta)=L_{A} \int_{0}^{\pi}|\sin (\alpha-\beta)| f_{\mathscr{R}}(\alpha) d \alpha,
$$

cf., e.g. Mecke and Stoyan (1980).

In a degraded case the intersection points are not observable. Instead of $P_{L}^{0}(\beta)$ we suggest the use of the scaled variogram of grey values

$$
V_{L}(d, \beta)=\frac{E|Z(x)-Z(y)|}{d},
$$
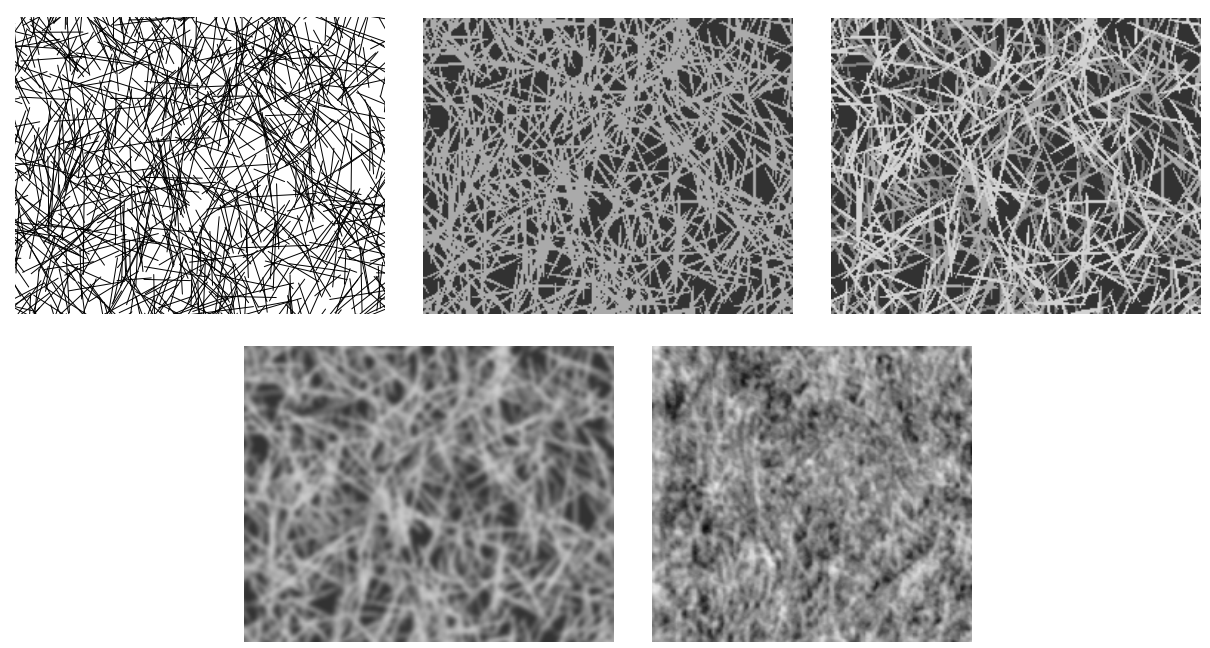

Fig. 1. Simulation from four different image models and an image of a paper sample (lower right). The size of the simulated images is $250 \times 200$. Upper left is a Boolean fibre model with intensity $\lambda=0.014$ and fibres equal to line segments with uniform length $l$ in $(20,60)$ and elliptic orientation distribution with parameters $(\tau, \kappa)=(1.603,0.888)$. Upper middle is the digital image of the corresponding 'thick' fibre model of ellipses (3) with $250 \times 200$ pixels. The minor axis of the ellipses is always 1. Upper right is the digital greyscale image of a realization of the corresponding dead leaves model with finite time. The background has grey value 50, the first 'layer' of fibres 130, the second 170 and the third 210, respectively. Lower left is the digital greyscale image obtained by additional blurring, using a moving average filter of size $(2 m+1) \times(2 m+1)$, with $m=1$, a total of four times. This approximates a Gaussian filter with $\sigma^{2}=4 / 3\left(m^{2}+m\right)$. Lower right is a digital greyscale image of size $216 \times 200$ pixels $(10.2 \mathrm{~mm} \times 9.4 \mathrm{~mm})$ from a layer of a paper sample. Resolution is $47 \mu \mathrm{m}$. 
where $x, y \in R^{2}$ are points on $L_{\beta}$ at the distance $d=$ $\|x-y\|$. In what follows, let the typical segment and its thick version be denoted by $\Gamma_{0}$ and $\Xi_{0}$, respectively, $c f$. (1) and (3). Then, under mild regularity conditions we have for a binary random field $\left\{Z(x)=1_{\Xi}(x)\right\}$ and small $d, c f$. Kärkkäinen et al. (2001a),

$$
V_{L}(d, \beta) \approx\left(1-d P_{L}^{0}(\beta) / 2\right) P_{L}^{1}(\beta),
$$

where $P_{L}^{1}(\beta)$ is the intensity of $L_{\beta} \cap \partial \Xi$. This intensity is related to $P_{L}^{0}(\beta)$ by (Kärkkäinen et al., 2001a;b; Molchanov and Stoyan, 1994)

$$
P_{L}^{1}(\beta)=\exp \left(-\lambda E v_{2}\left(\Xi_{0}\right)\right) 2 \frac{E h_{\beta}\left(\Xi_{0}\right)}{E h_{\beta}\left(\Gamma_{0}\right)} P_{L}^{0}(\beta),
$$

where $v_{2}$ denotes the two-dimensional Lebesgue measure. If

$$
\frac{E h_{\beta}\left(\Xi_{0}\right)}{E h_{\beta}\left(\Gamma_{0}\right)} \approx 1,
$$

meaning that the thickness of fibres is small compared to the length of fibres, then approximately for small $d$

$$
V_{L}(d, \beta) \approx \xi\left(1-d P_{L}^{0}(\beta) / 2\right) P_{L}^{0}(\beta),
$$

where $\xi=2 \exp \left(-\lambda E v_{2}\left(\Xi_{0}\right)\right)$. For sufficiently small $d,\left(1-d P_{L}^{0}(\beta) / 2\right) \approx 1$ and thus $V_{L}(d, \beta) \approx \xi P_{L}^{0}(\beta)$. The results can be extended to a dead leaves model, $c f$. Jeulin (2000). However, note that $\xi$ is not any more the same as in (9) because the dead leaves model concerns multicoloured images.

In practice, the scaled variogram (5) is observed from a digital image $\{z(l, m): l, m$ integers $\}$ along a digital line $\bar{L}_{\beta}$ chosen to be a pixel sequence of 8connected pixels in different directions $\beta$. When the pixels $(l, m),\left(l^{\prime}, m^{\prime}\right)$ are neighbours, the distance $d=$ $\left\|(l, m)-\left(l^{\prime}, m^{\prime}\right)\right\|$ is either 1 for horizontal and vertical neighbours, or $\sqrt{2}$ for the diagonal ones. We use the estimator

$$
\hat{V}_{L}(\beta)=\frac{\sum_{(l, m),\left(l^{\prime}, m^{\prime}\right) \in \bar{L}_{\beta}}\left|z(l, m)-z\left(l^{\prime}, m^{\prime}\right)\right|}{\sum_{(l, m),\left(l^{\prime}, m^{\prime}\right) \in \bar{L}_{\beta}}||(l, m)-\left(l^{\prime}, m^{\prime}\right) \|},
$$

\section{cf. Kärkkäinen et al. (2001a;b).}

We consider 8 directions. The main directions $\beta_{1}=0, \beta_{3}=\pi / 4=0.785, \beta_{5}=\pi / 2=1.571, \beta_{7}=$ $3 \pi / 4=2.356$ with distances between neighbour pixels $d_{1}=1, d_{3}=\sqrt{2}, d_{5}=1, d_{7}=\sqrt{2}$, respectively, and the intermediate directions $\beta_{2}=0.464, \beta_{4}=1.107, \beta_{6}=$ $2.034, \beta_{8}=2.678$. Let us define

$$
F_{i}(\theta)=\int_{0}^{\pi}\left|\sin \left(\alpha-\beta_{i}\right)\right| f_{\mathscr{R}}(\alpha ; \theta) d \alpha
$$

in all directions while

$$
G_{i}\left(\theta, L_{A}\right)=F_{i}(\theta)\left[1-d_{i} L_{A} F_{i}(\theta) / 2\right]
$$

holds for $i=1,3,5,7$ and

$$
\begin{aligned}
G_{i}\left(\theta, L_{A}\right)= & \frac{d_{i-1}}{d_{i-1}+d_{i+1}} G_{i-1}\left(\theta, L_{A}\right) \\
& +\frac{d_{i+1}}{d_{i-1}+d_{i+1}} G_{i+1}\left(\theta, L_{A}\right)
\end{aligned}
$$

for $i=2,4,6,8$. Then according to Kärkkäinen et al. (2001a)

$$
E \hat{V}_{L}\left(\beta_{i}\right) \approx \xi L_{A} G_{i}\left(\theta, L_{A}\right)
$$

for $i=1, \ldots, 8$.

In the estimation of the parameters $\theta=(\tau, \kappa)$, using these eight directions, we can eliminate the unknown $\xi$ and use a least square estimation procedure. It leads to numerical minimization of the sum of weighted squares, compare to Kärkkäinen et al. (2001b),

$\hat{\chi}\left(\theta, L_{A}\right)=\sum_{i=2}^{8} W_{i}\left[\hat{V}_{L}\left(\beta_{i}\right)-\hat{V}_{L}\left(\beta_{1}\right) \frac{G_{i}\left(\theta, L_{A}\right)}{G_{1}\left(\theta, L_{A}\right)}\right]^{2}$,

where $W_{i}=L_{i} L_{1} /\left(L_{i}+L_{1}\right)$ for $i=2, \ldots, 8, L_{i}$ is the total length of the sampling line in direction $\beta_{i}$. In the ideal situation where $P_{L}^{0}(\beta)$ can be estimated the formula (Kärkkäinen et al., 2001b)

$$
\hat{\chi}(\theta)=\sum_{i=2}^{8} W_{i}\left[\hat{P}_{L}^{0}\left(\beta_{i}\right)-\hat{P}_{L}^{0}\left(\beta_{1}\right) \frac{F_{i}(\theta)}{F_{1}(\theta)}\right]^{2}
$$

should be used instead of (15). Note that in a general use of the formula (16), the directions and the number of sampling lines can differ from the collection $\left\{\beta_{i}, i=\right.$ $1, \ldots, 8\}$.

\section{RESULTS AND PERSPECTIVES}

We have estimated the orientation parameters of the elliptic distribution using data from each of the five images shown in Fig. 1. For the image of line segments (upper left of Fig. 1), the data consist of estimated point intensities $\hat{P}_{L}^{0}\left(\beta_{i}\right)$. For the binary $(0,1)$-valued image, the dead leaves simulation, the blurred image and the paper image, the data are scaled variograms $\hat{V}_{L_{\text {bin }}}\left(\beta_{i}\right), \hat{V}_{L_{d l}}\left(\beta_{i}\right), \hat{V}_{L_{\text {blur }}}\left(\beta_{i}\right)$ and $\hat{V}_{L_{\text {paper }}}\left(\beta_{i}\right)$, respectively, calculated from (10). The data are shown in Table 1 , together with $\hat{\tau}$ and $\hat{\kappa}$ obtained from (16) for point intensities and from (15) for scaled variograms. From 
the paper image in Fig. 1 the estimated parameters are $(\hat{\tau}, \hat{\kappa})=(1.603,0.888)$, the same values used in simulation of the four other images. In the cases of simulated data the estimates obtained from scaled variograms should be compared with the ones obtained from the estimated point intensities. The estimates are quite similar except in the blurred case where $\hat{\kappa}$ is somewhat smaller. The ratios $\hat{V}_{L_{b l u r}}\left(\beta_{i}\right) / \hat{V}_{L_{d l}}\left(\beta_{i}\right)$ show a diagonal effect which partly explains the differences in the estimates.

We have also estimated the parameters, using the simplified relation (Kärkkäinen et al., 2001b)

$$
V_{L}(d, \beta) \approx \xi P_{L}^{0}(\beta)
$$

compared with (9). Quite similar estimates were obtained in this example. With more pronounced anisotropy or larger $d$ it might, however, be important to use the refined relation (9).

\section{ACKNOWLEDGEMENTS}

This work has been supported by Academy of Finland (MaDaMe-project No 50139), INTAS-project No 0537 and NorFA-project No 000519. The authors also thank professors Antti Penttinen, Nikolai G. Ushakov, Alexandra P. Ushakova and Dominique Jeulin for cooperation.

Table 1. In eight different directions $\beta_{i}$ theoretical and estimated point intensities $P_{L}^{0}\left(\beta_{i}\right), \hat{P}_{L}^{0}\left(\beta_{i}\right)$ and scaled variograms $\hat{V}_{L_{\text {bin }}}\left(\beta_{i}\right), \hat{V}_{L_{d l}}\left(\beta_{i}\right), \hat{V}_{L_{\text {blur }}}\left(\beta_{i}\right), \hat{V}_{L_{\text {paper }}}\left(\beta_{i}\right)$ are shown for the binary, the dead leaves, the blurred and the paper image of Fig. 1, respectively. Estimates of $\tau$ and $\kappa$ are also shown.

\begin{tabular}{lllllll}
\hline$\beta_{i}$ & $P_{L}^{0}\left(\beta_{i}\right)$ & $\hat{P}_{L}^{0}\left(\beta_{i}\right)$ & $\hat{V}_{L_{\text {bin }}}\left(\beta_{i}\right)$ & $\hat{V}_{L_{d l}}\left(\beta_{i}\right)$ & $\hat{V}_{L_{\text {blur }}}\left(\beta_{i}\right)$ & $\hat{V}_{L_{\text {paper }}}\left(\beta_{i}\right)$ \\
\hline 0.000 & 0.401 & 0.419 & 0.266 & 38.51 & 8.072 & 1.573 \\
0.464 & 0.388 & 0.406 & 0.239 & 35.04 & 7.753 & 10.745 \\
0.785 & 0.364 & 0.377 & 0.223 & 32.80 & 7.522 & 10.163 \\
1.107 & 0.335 & 0.346 & 0.220 & 32.52 & 7.298 & 9.697 \\
1.571 & 0.311 & 0.320 & 0.217 & 32.07 & 6.988 & 9.262 \\
2.034 & 0.330 & 0.334 & 0.217 & 31.93 & 7.214 & 9.599 \\
2.356 & 0.358 & 0.359 & 0.216 & 31.79 & 7.378 & 10.036 \\
2.678 & 0.384 & 0.390 & 0.237 & 34.64 & 7.668 & 10.666 \\
\hline$\hat{\tau}$ & 1.603 & 1.655 & 1.658 & 1.671 & 1.639 & 1.603 \\
$\hat{\kappa}$ & 0.887 & 0.910 & 0.901 & 0.876 & 0.773 & 0.888 \\
\hline
\end{tabular}

\section{REFERENCES}

Erkkilä A-L, Pakarinen P, Odell M (1998). Sheet forming studies using layered orientation analysis. Pulp and Paper Canada 99:T39-T43.

Forgacs OL, Strelis J (1963). The measurement of the quantity and orientation of chemical pulp fibres in the surfaces of newsprint. Pulp and Paper Mag. of Canada 64:T3-T13.

Glasbey CA, Horgan GW (1995). Image Analysis for the Biological Sciences. Chichester: Wiley.

Hutten IM (1994). Paper machine evaluation by fiber orientation profile analysis. Tappi Journal 77:187-192.

Jeulin D (1989). Morphological modeling of images by sequential random functions. Signal Processing $16: 403-431$.

Jeulin D (2000). Variograms of the dead leaves model. Research Report N-31/00/MM, Paris School of Mines Publication.

Kärkkäinen S, Jensen EBV, Jeulin D (2001a). On the orientational analysis of Boolean fibres from digital images. Research Report 15, Laboratory for Computational Stochastics, Department of Mathematical Sciences, University of Aarhus. Submitted.

Kärkkäinen S, Penttinen A, Ushakov NG, Ushakova AP (2001b). Estimation of orientation characteristic of fibrous material. Adv Appl Prob 33:559-575.

Matheron G (1972). Ensembles fermés aléatoires, ensembles semi-Markoviens et polyèdres poissoniens. Adv Appl Prob 4:508-41.

Mecke J, Stoyan D (1980). Formulas for stationary planar fibre processes I - general theory. Math Operationsforsch Statist, Ser Stat 11:267-79.

Molchanov I, Stoyan D (1994). Directional analysis of fibre processes related to Boolean models. Metrika 41:183199

Paakkari T, Serimaa R, Hattula T, Ahtee M (1984). Determination of fibre orientation in a paper sample using x-ray diffraction. Paperi Ja Puu 66: 569-75.

Serra J (1982). Image Analysis and Mathematical Morphology. London: Academic Press. 